\title{
Plasticity in the Organization of Adult Cerebral Cortical Maps: A Computer Simulation Based on Neuronal Group Selection
}

\author{
John C. Pearson, Leif H. Finkel, and Gerald M. Edelman \\ The Neurosciences Institute and The Rockefeller University, New York, New York 10021
}

Recent experimental evidence from the somatosensory, auditory, and visual systems documents the existence of functional plasticity in topographic map organization in adult animals. This evidence suggests that an ongoing competitive organizing process controls the locations of map borders and the receptive field properties of neurons. A computer model based on the process of neuronal group selection has been constructed that accounts for reported results on map plasticity in somatosensory cortex.

The simulations construct a network of locally connected excitatory and inhibitory cells that receives topographic projections from 2 receptor sheets corresponding to the glabrous and dorsal surfaces of the hand (a typical simulation involves approximately 1500 cells, 70,000 intrinsic and 100,000 extrinsic connections). Both intrinsic and extrinsic connections undergo activity-dependent modifications according to a synaptic rule based on heterosynaptic interactions.

Repeated stimulation of the receptor sheet resulted in the formation of neuronal groups-local sets of strongly interconnected neurons in the network. Cells in most groups were found to have similar receptive fields: they were exclusively glabrous or dorsal despite equal numbers of anatomical connections from both surfaces. The sharpness of map borders was due to the sharpness of the underlying group structure; shifts in the locations of these borders resulted from competition between groups.

Following perturbations of the input, the network underwent changes similar to those observed experimentally in monkey somatosensory cortex. Repeated local tapping on the receptor sheet resulted in a large increase in the magnification factor of the stimulated region. Transection of the connections from a glabrous region resulted in the organization of a new representation of corresponding dorsal region. The detailed simulations provide several insights into the mechanisms of such changes, as well as a series of predictions about cortical behavior for further experimental test.

One of the long-standing problems in neuroscience has been to determine the principles underlying the formation and maintenance of ordered topographic maps. The prevailing view has

\footnotetext{
Received Mar. 17, 1987; revised July 9, 1987; accepted July 21, 1987.

This work was supported by grants from the International Business Machines Corporation, Johnson and Johnson, and Senator Jacob Javits Center for Excellence in Neuroscience Award NS-22789. We thank Dr. George N. Reeke, Jr. for advice and extensive help in implementation of the simulations.

Correspondence should be addressed to Gerald M. Edelman, Rockefeller University, Box 287, 1230 York Avenue, New York, NY 10021.

Copyright (C) 1987 Society for Neuroscience 0270-6474/87/124209-15\$02.00/0
}

been that these maps, once developmentally established, remain fixed for the duration of an animal's lifetime. Early observations suggested the possibility, however, that certain cortical maps may be labile (Leyton and Sherrington, 1917). More recently, evidence has emerged that maps in the somatosensory cortex continually reorganize, even in adult animals (Kaas et al., 1983). This evidence suggests that the functional or physiologically recorded map may be related to the underlying anatomical map through complex dynamic processes. Such processes are likely to be involved inasmuch as the presence of divergent and overlapping arborizations in the underlying cortical anatomy allow for a large degree of possible variability in the receptive field map. Given the anatomical constraints, it is difficult to understand how single cortical cells acting independently could generate somatotopic maps with sharp but movable borders. The observed plasticity of such maps can be accounted for by the presence of local groups of functionally interactive cells that act cooperatively to yield map organization. Competition between such neuronal groups could give rise to continuous map borders that could shift dynamically upon changes in input.

In an effort toward understanding these complex processes, we present here a detailed model of the dynamic control of map organization. This model, which is based on the theory of neuronal group selection (Edelman, 1978), is chiefly concerned with the functional properties of synaptic populations undergoing modifications within a fixed network anatomy during various forms of sensory stimulation. In developing this model for computer simulation, we deliberately restricted ourselves to the simplest network that would yield insights into synaptic selection in maps. This minimal model, while explicitly detailed, does not incorporate features such as multiple ascending synaptic levels, interconnected cortical areas, and multiple cell types, and therefore does not strictly simulate real cortex. The structure chosen was nonetheless based on realistic anatomical and physiological assumptions and allowed us to investigate (1) factors determining the receptive field of a cell, (2) principles controlling the magnification factor of a representation, (3) determinants of the locations of map borders, and (4) relative contributions to map plasticity of changes in synaptic strengths of extrinsic versus intrinsic connections.

Although several of the results have general application, our empirical reference in this paper is specifically to the somatosensory cortex of adult monkeys (Mountcastle, 1957) and the plasticity of cortical somatolopic maps (Kaas et al., 1983). Map changes quite similar to those with which we will be concerned have also been observed in adult animals of a number of species and in several sensory modalities at both cortical and subcortical levels (see Mountcastle, 1984, for review).

Areas $3 \mathrm{~b}$ and 1 of owl and squirrel monkey cortex each contain 


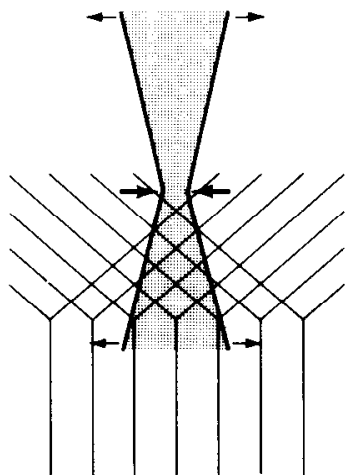

Group Confinement

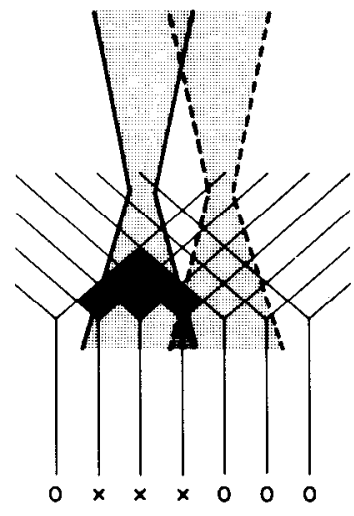

Group Selection

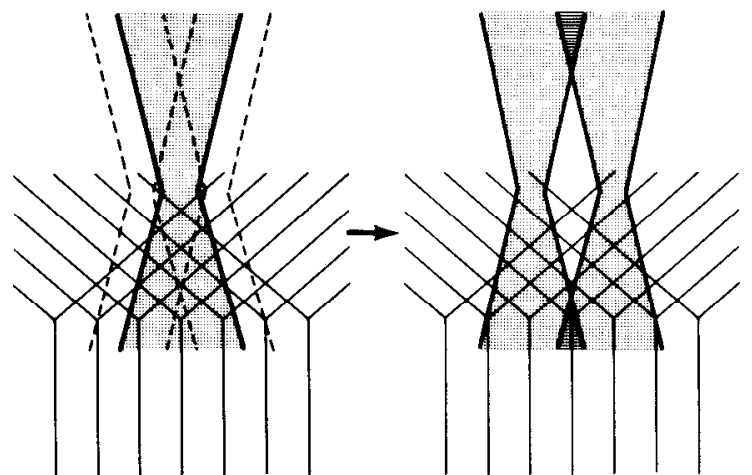

Group Competition

Figure 1. Schematic representation of neuronal group processes in a qualitative model of map organization. Hourglass figures represent outlines of neuronal groups extending through all cortical laminae (waist of hourglass is layer IV); ascending Y-shaped figures represent outlines of divergent, overlapping thalamocortical afferents. Group confinement is a property of the intrinsic cortical connectivity which restricts activity to local domains, allowing groups to form. Group selection requires coactive extrinsic inputs, which strengthen the intrinsic connections of a group through synaptic modifications following a particular synaptic rule. The $\times$ 's denote coactive afferents, the o's denote uncorrelated afferents. The blackened region receives maximum coactive and minimum uncorrelated input. This leads, through a synaptic modification mechanism dependent on coactive inputs, to selection of the leftmost of the 2 groups. Group competition controls which cells belong to which groups and also regulates the overlap of the receptive fields of adjacent groups. In the hypothetical case shown here, competition between 3 groups leads to the complete dissolution of the central one. Such competition is effected through the synaptic rule and depends upon the balance of stimulation received by the various groups (from Edelman and Finkel, 1984).

a complete somatotopic representation of the body surface; cells in these areas respond to light touch (Merzenich et al., 1978; Dykes, 1983; Whitsel and Kelly, 1986). Detailed microelectrode penetrations (Merzenich et al., 1983a) reveal that these maps are dynamic: under normal circumstances there are marked shifts over time in map borders between body parts. More dramatic changes are seen after perturbation of the input. For example, if one or more fingers are amputated, if a cutaneous nerve is transected, or if a region is repeatedly stimulated for prolonged periods, map borders can move hundreds of microns, and entirely new representations can emerge (Merzenich et al., 1983a, b; Jenkins et al., 1984).

To account for these results, we have previously described a qualitative model (Edelman and Finkel, 1984) of cortical map organization based on the theory of neuronal group selection (Edelman, 1978, 1981). This theory proposes that the nervous system operates as a selective system, similar in some respects to the operation of natural selection on organisms. In the nervous system, however, the selection takes place by specific neural mechanisms involved in the development of connectivity and experience-dependent synaptic modifications. The population proposed to be undergoing selection consists of variant neuronal groups - local sets of approximately 500-1500 strongly interconnected neurons. The cells in a group act collectively in determining their receptive field properties, and thus groups serve as the basic units of map organization.

Neuronal groups are not built-in anatomical structures, but functional units whose membership is determined by synaptic strengths. A set of 3 heuristic processes that function in parallel to govern group behavior has been proposed (Fig. 1; Edelman and Finkel, 1984). Groups are assumed to arise through a process of group confinement, an intrinsic cortical process that depends upon the patterns of activity flow in the cortex, the balance of excitation and inhibition in different laminae, and local variabilities in connectivity. The second process of group selection involves differential changes in the strengths of syn- aptic connections based on correlated inputs. Only the presumptive groups that are most strongly activated strengthen and refine their receptive fields. Once selected, a process of competition between groups over cell membership and receptive field properties is responsible for the reorganizations seen in adult maps.

This model, depicted in Figure 1, provides only a general qualitative scheme for understanding maps in terms of population variables. In order to establish that such variables could account in mechanistic detail for the experimentally observed properties of map plasticity, we have instantiated them in the present study, which employs detailed computer simulations. Using this synthetic approach to neural modeling, we show explicitly that neuronal groups can be formed in an initially unorganized network and that a network organized into such groups exhibits an ordered topographic map with many of the observed properties of cortical maps. This model was subjected to stringent computer tests, providing the opportunity to determine in detail the effect of various perturbations upon map reorganization.

\section{Materials and Methods}

Anatomical connectivity. The computer model simulates a 2-dimensional network of interconnected excitatory (e-cells) and inhibitory (i-cells) neurons and an input array of sensory receptors. The cells are uniformly spread over a rectangular grid. All simulations reported here used a $32 \times 16$ grid with a total of $1024 \mathrm{c}$-cclls and $512 \mathrm{i}$-cells, i.e., 2 e-cells and 1 i-cell at each of $512(=32 \times 16)$ grid points. The e-cell to $\mathrm{i}$-cell ratio is not a critical factor in network behavior and was chosen for convenience since receptive field measurements are primarily made on e-cells.

Two identical sensory receptor arrays, corresponding to the glabrous and dorsal surfaces of the hand, are topographically mapped onto the network (Fig. 2). Regions corresponding to 4 fingers and a subjacent palm are present, and the 2 arrays are joined along the border of digit 1. Each array contains 512 receptors with $96(=8 \times 12)$ glabrous and 96 dorsal receptors on each finger, and $128(=32 \times 4)$ receptors in the glabrous and dorsal subphalangeal regions. Instructions in the program's input can create and move a 2 -dimensional stimulus about the receptor 
Table 1. Number of connections received by a cell as a function of distance

\begin{tabular}{|c|c|c|c|c|c|c|}
\hline \multirow[b]{2}{*}{ Ring } & \multicolumn{6}{|c|}{ Number of connections/cell } \\
\hline & $\mathrm{e} \rightarrow \mathrm{e}$ & $\mathrm{i} \rightarrow \mathrm{e}$ & $\mathrm{r} \rightarrow \mathrm{e}$ & $\mathrm{e} \rightarrow \mathrm{i}$ & $\mathrm{i} \rightarrow \mathrm{i}$ & $\mathbf{r} \rightarrow \mathrm{i}$ \\
\hline 0 & 1 & 1 & 2 & 1 & 0 & 0 \\
\hline 1 & 8 & 8 & 16 & 4 & 0 & 0 \\
\hline 2 & 8 & 8 & 32 & 8 & 0 & 0 \\
\hline 3 & 0 & 0 & 48 & 24 & 0 & 0 \\
\hline 4 & 0 & 0 & 0 & 32 & 0 & 0 \\
\hline
\end{tabular}

The excitatory (e) cells and inhibitory (i) cells in the network receive connections from each other and from receptors $(r)$ in an input array (e.g., $\mathrm{e} \rightarrow \mathrm{i}$ means an input to an inhibitory cell from an excitatory cell). The number of connections each cell receives from each cell type and from receptors is specified as a function of distance or "ring" (see Fig. 3). The number of $r \rightarrow$ e connections listed are from both glabrous and dorsal receptor arrays, each array contributes an equal number of connections per cell.

sheet, activating those receptors it covers (the stimulus can be any size or shape; we typically use a $3 \times 3$ solid square). The effect of the receptors on the cells of the network is exclusively excitatory.

Parameters of the receptor-cell connection scheme detailed in Figure 3 are given in Table 1: Each e-cell receives connections of equal density from the $7 \times 7$ region centered around the topographically corresponding receptor in both the glabrous and dorsal receptor arrays; i-cells do not receive any extrinsic input. Each e-cell receives 49 glabrous and 49 dorsal inputs, for a total of $100,352 \mathrm{r} \rightarrow$ e connections. Arborizations of fibers from nearby receptors overlap extensively in the network; the regions of external input for adjacent cells overlap by $86 \%$ on average.

There are 4 classes of intrinsic connections within the network, denoted by $\mathrm{e} \rightarrow \mathrm{e}, \mathrm{i} \rightarrow \mathrm{e}, \mathrm{e} \rightarrow \mathrm{i}$, and $\mathrm{i} \rightarrow \mathrm{i}$, where $\mathrm{e}$ and $\mathrm{i}$ represent e-cells and i-cells, respectively. All excitatory synapses are assumed to be within one length constant on the distal dendritic tree; all inhibitory inputs are assumed to be on the proximal dendrite and act to shunt the potentials produced more distally. The number of connections of each class received by a cell is specified according to the distance between the preand postsynaptic elements (see Table 1, Fig. 3). The choice of which particular cells are connected is decided randomly; multiple connections are allowed but self-connections are not.

All cells in the network receive the same number of synapses, but near the edges, those connections that would have extended outside the network border are made instead to the cell located inside the network at the mirror image of the original target cell. Thus, the density of connections is higher near the borders of the network. As discussed below, these boundary conditions affect the population dynamics of the network.

The scheme of connections was constrained by considerations of scale and was arrived at empirically through a series of tests checking for network stability under a range of input conditions. The scheme of intrinsic connections is essentially one of local excitation and lateral inhibition (Fig. $3 B$ ): e-cells excite nearby e-cells and slightly more distant i-cells; i-cells inhibit nearby e-cells. The effect of activating a local population of e-cells is a build-up of activity in that area followed by inhibition of a larger concentric region.

Neuronal properties. Each neuron in the network is characterized by a number of parameters (Table 2) that control the response of the cell to input stimulation. At each time step, the voltage of each cell is determined based upon the voltage of the cell during the previous time step and upon the excitatory and inhibitory inputs received by the cell during that time step. The output, $s$, of each is cell is then determined as a sigmoidal function of the voltage, $v$, as defined in equation (1):

$$
s_{i}=\sigma\left(v_{i}, \theta_{s}, \epsilon_{s}\right)=\left[\exp -\left(v_{i}-\theta_{s}\right) / \epsilon_{s}+1\right]^{-1}
$$

where $\theta_{s}$ and $\epsilon_{s}$ are parameters determining the inflection point and the nonlinearity, respectively, of the sigmoidal function, $\sigma$. For small voltages $\left(v_{i}<0.2 \theta_{s}\right) s_{i}$ is set to zero. The output is a continuous function, corresponding to the firing rate of a single neuron or the instantaneous averaged firing of a local ensemble of neurons. The voltage, $v$, undergoes exponential decay $\left(\right.$ rate $\delta_{v}$ ) and is increased by the sum of the input from c-cells and receptors multiplicd by 2 dynamic shunting functions, $\sigma_{l}$ and $\sigma_{v}:$

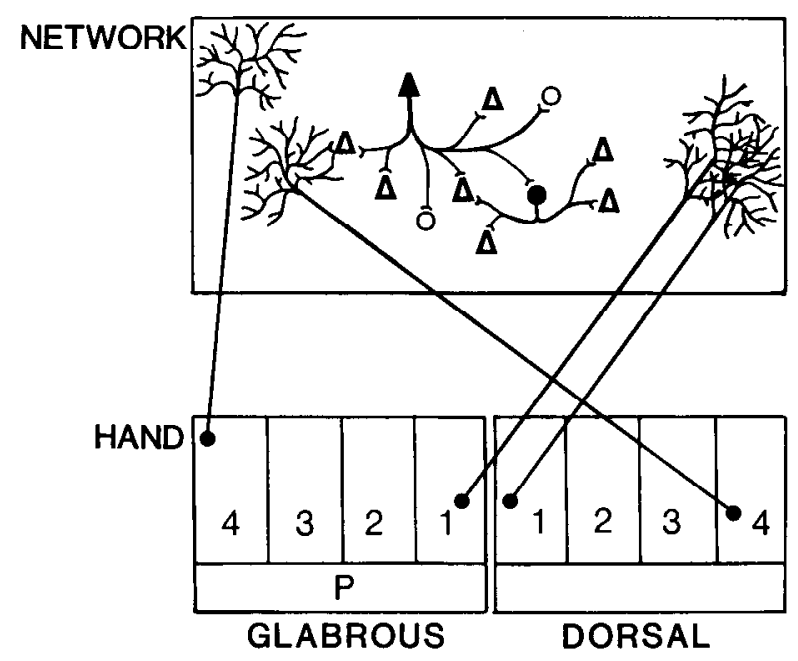

Figure 2. Schematic diagram of the basic elements and architecture of the model. The network consists of a 2-dimensional array of both excitatory cells (triangles) and inhibitory cells (circles). The intrinsic connections are characterized by local excitation and lateral inhibition. Excitatory cells receive connections from nearby excitatory and inhibitory cells, whereas inhibitory cells receive connections from relatively more distant excitatory cells. Excitatory cells also receive excitatory connections from 2 external arrays of receptors, corresponding to the glabrous and dorsal regions of the hand. The arrays contain regions corresponding to digits $1-4$ and the palm $(P)$, and they are joined along the border of digit 1 . These regions are defined by convention only; there are no anatomical boundaries on the arrays. Arborizations from nearby receptors overlap extensively in the network, and each array projects topographically onto the network in such a fashion that corresponding glabrous and dorsal regions project to the same network location.

$$
\begin{aligned}
v_{i}(t+1)= & \left(1-\delta_{v}\right) v_{i}(t)+\sigma_{I} \sigma_{v}\left[\kappa_{E} \sum_{j \in E} \eta_{i j}(t) S_{j}(t)\right. \\
& \left.+\kappa_{R} \sum_{j \in R} \eta_{i j}(t) r_{j}(t+1)\right]
\end{aligned}
$$

where

$$
\sigma_{I}=\sigma\left(\sum_{j \epsilon l} s_{j}(t), \theta_{l},-\epsilon_{l}\right), \quad \sigma_{v}=\sigma\left[v_{i}(t), \theta_{v},-\epsilon_{v}\right]
$$

$\sigma_{\text {, }}$ simulates shunting inhibition and $\sigma_{v}$ simulates synaptic saturationthe decrease in transmembrane ionic driving force with increase in $v . \eta$ is the postsynaptic strength, which for e-cells is plastic as defined below. $r_{j}$ is the activity of receptor $j$, and is 0 or 1 , signaling the absence $(0)$ or presence (1) of a stimulus at that point on the hand. The definitions and values of the parameters in this paper are listed in Table 2. Equation (2) is schematically illustrated in Figure 4.

Shunting inhibition, in the form of a nonnegative multiplicative term, was used instead of hyperpolarizing inhibition, a subtractive term, because of the greater dynamic stability it conferred on the network. The excitatory input potentials to a cell are reduced to zero when a sufficiently large number of inhibitory cells fires. This kind of inhibition can always balance the excitatory processes and thus return the network to equilibrium after periods of intense activation. As discussed below, this is especially important in networks with synaptic plasticity properties that tend to strengthen excitatory synapses.

Synaptic plasticity rules. The rule for synaptic plasticity used here is based on known properties of hippocampal (Wigstrom and Gustafsson, 1983; Kelso et al., 1986; Malinow and Miller, 1986), cerebellar (Ito et al., 1982), and a variety of invertebrate synapses (Hawkins et al., 1983; Huganir et al., 1986), and is adapted from the dual rules model (Finkel, 1985; Finkel and Edelman, 1985, 1987). In this model, local biochemical modifications of the channels or receptors at a synapse are governed by the spatial and temporal pattern of heterosynaptic inputs to the neuron. The main assumption, which is supported by a growing body of experimental evidence (reviewed in Finkel and Edelman, 1987), is 

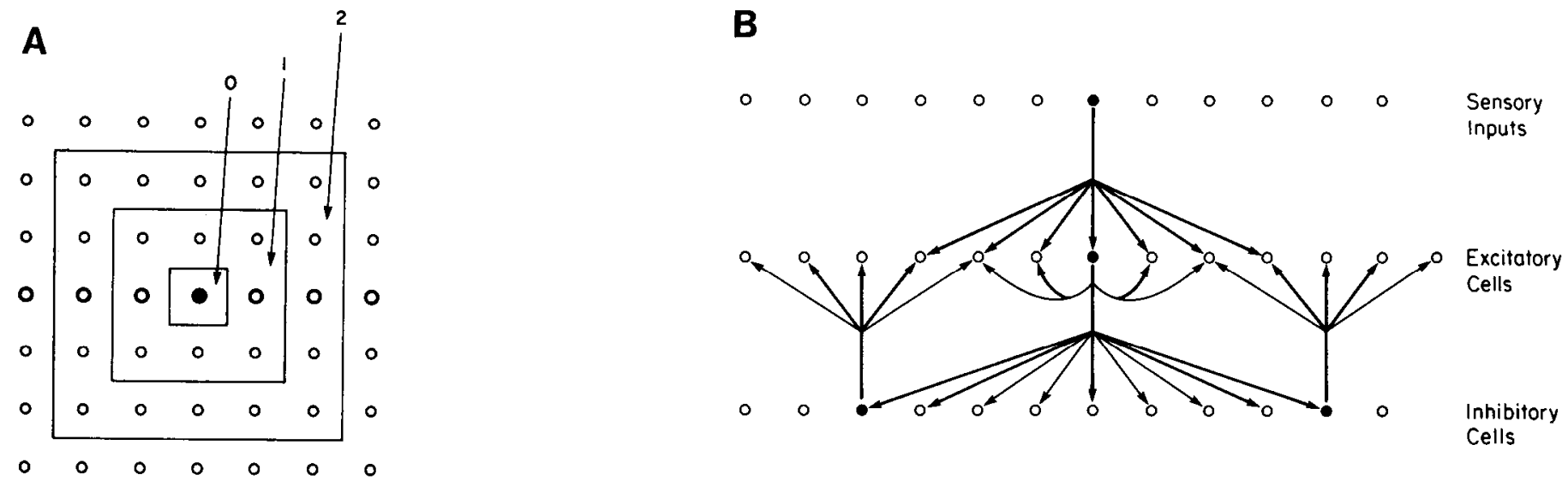

Figure 3. Cell-cell connection scheme. $A$, Depiction of the square "rings" (0th, 1st, and $2 \mathrm{nd}$ ) used to assign connections (only a small part of the network is shown) as a function of distance between cells (small circles). The rings shown here are those of the cell marked with the solid circle. Each cell in the network has a similar set of rings constructed about it for the purpose of assigning connections. Every cell within a given ring is considered to be the same distance from the cell in the center (0th ring). An identical construction is used to assign the extrinsic connections from the receptor array to the network. The line of thicker circles corresponds to the row of cells whose connections are depicted in $B$. B. Schematic of a 1-dimensional section through the network showing the spatial distribution of excitatory and inhibitory connections. The output connections of the cells marked with solid circles are drawn. The thick and thin lines represent high- and low-density connections, respectively (Table 1). Corresponding to each of the 1024 grid points there is 1 glabrous receptor, 2 e-cells, and $1 \mathrm{i}$-cell. Only 1 e-cell per grid point is drawn.

that the modifiability of a channel or receptor depends upon its functional state (e.g., open, closed, inactivated).

In the present model, a simplified version of this postsynaptic rule is used and only the $\mathrm{e} \rightarrow \mathrm{e}$ and $\mathrm{r} \rightarrow \mathrm{e}$ connections are plastic. Each plastic postsynaptic terminal contains an equal number of channels, each of which is assumed to have 4 conducting states, $\mathrm{O}, \mathrm{P}, \mathrm{O}^{*}$, and $\mathrm{P}^{*}$ (Fig. $5 A$ ). The $\mathrm{O}$ (open) and $\mathrm{P}$ (plastic) states have ionic conductance, $g$; the corresponding modified states, $\mathrm{O}^{*}$ and $\mathrm{P}^{*}$, have conductance, $g^{*}$ (where $g^{*}>g$ ). Only channels in the P states are modifiable. The net synaptic strength, $\eta$, defined as the conversion factor between presynaptic activity and postsynaptic potential, depends upon the fraction of the channels in each conducting state, as defined in equation (3), where $o, p, o^{*}$, and $p^{*}$ are the fraction of channels in each respective state (i.e., $o+p+$ $\left.o^{*}+p^{*}=1\right)$.

$$
\eta=g(o+p)+g^{*}\left(o^{*}+p^{*}\right)
$$

The postsynaptic rule can, in general, be applied to multiple channel states-open, closed, or inactivated (Finkel and Edelman, 1985). In this simplified version, we have subsumed the closed channel states into the closed receptor state $\left(r_{j}-s_{j}-0\right.$ in equation 2$)$.

The channels undergo state transitions as illustrated in Figure $5 A$, and these transitions are the basis for synaptic plasticity. The transition rate from $\mathrm{O} \rightarrow \mathrm{P}$ is an increasing sigmoidal function of voltage, while the backward transition from $P \rightarrow O$ is an exponential decay. Thus, the larger the voltage in the postsynaptic cell, the larger the $\mathrm{P} / \mathrm{O}$ ratio. The transitions between the modified channels are the same except for different parameter values. Note that such transitions do not change the synaptic strength.

On the other hand, transitions from $\mathrm{P} \rightarrow \mathrm{P}^{*}$ strengthen the synapse and transitions from $\mathrm{P}^{*} \rightarrow \mathrm{P}$ weaken it. The forward transition rate from $\mathrm{P} \rightarrow \mathrm{P} *$ is controlled by the amount of modifying substance, $m_{i j}$, present at the postsynaptic terminal:

$$
m_{i j}(t+1)=\kappa_{m} s_{j}-\delta_{m} m_{i j}(t)(0 \leq m \leq 1)
$$

As shown in equation (4) $m(t)$ grows at a rate $\left(\kappa_{m}\right)$ proportional to the activity of the presynaptic cell, $s_{j}$, and exponentially decays (rate $\delta_{m}$ ) with time (see Table 2 for parameter values). The backward transition from $\mathrm{P}^{*} \rightarrow \mathrm{P}$ is a $\mathrm{P}^{*}$ independent decay.

The net result of these transitions is expressed in equations 5-8, with parameters defined in Table 2 and $\sigma_{o p}=\sigma\left[v(t), \theta_{o p}, \epsilon_{o p}\right], \sigma_{o p}{ }^{*}=\sigma[v(t)$, $\left.\theta_{o p}^{*}, \epsilon_{o p}^{*}\right]$ :

$$
\begin{aligned}
o(t+1) & =o(t)\left(1-\kappa_{o p} \sigma_{o p}\right)+\delta_{p d} p(t) \\
o^{*}(t+1) & =o^{*}(t)\left(1-\kappa_{o p}{ }^{*} \sigma_{o p}{ }^{*}\right)+\delta_{p o}{ }^{*} p^{*}(t) \\
p(t+1) & =p(t)\left[1-\delta_{p o}-\kappa_{m} m(t)^{4}\right]+\kappa_{o p} \sigma_{o p} o(t) \\
& +\min \left(\delta_{p}, p^{*}\right)
\end{aligned}
$$

$$
\begin{aligned}
p^{*}(t+1)= & p^{*}(t)\left[1-\delta_{p o}{ }^{*}-\min \left(\delta_{p}, p^{*}\right)\right] \\
& +\kappa_{m} m(t)^{4} p(t)+\kappa_{o p}{ }^{*} \sigma_{o p}{ }^{*} o^{*}(t)
\end{aligned}
$$

To strengthen a synapse with this rule requires the conjunction of 2 events: First, the postsynaptic voltage must have risen high enough, relative to $\theta_{o p}$, that a significant fraction of the unmodified channels have made the transition to the $P$ state; second, the modifying substance, $m$, must have grown to near its maximum value of 1 (since the modification is proportional to $\mathrm{m}^{4}$ ). To weaken a synapse with this rule requires the conjunction of low $m$ and high voltage (relative to $\theta_{o p}{ }^{*}$ ). The time window for the conjunction of these 2 events is determined by the growth and decay rates of the state transitions and the production of modifying substance (Finkel and Edelman, 1985). These synaptic relationships are demonstrated in the simple simulation shown in Figure $5 B$

Program operation. The simulations were performed on IBM 4331 and 3090 computers. Programs were written in FORTRAN with extensive use of assembler utility routines (Reeke, 1984). Large simulations required $\sim 3.5$ megabytes of memory and $\sim 80$ min of CPU time on the 3090.

The receptive fields of all cells in the network could be determined simultaneously. This was accomplished by stimulating each point of the input array (in sequence) with a stimulus of a given size (typically $3 \times 3$ ) and recording the average response of each cell in the network during the stimulation of each such point (the stimulus was typically "on" for 3 steps and "off" for 7 steps). Note that the receptive fields were measured and not simply computed according to some rule.

Analysis of receptive fields and connection strengths was performed with a separate program and displayed on an IBM 5080 graphics terminal. This program can display many different features of the data, such as anatomical connections, connection strengths, individual receptive fields or receptive field maps, receptive field overlaps, etc., and was an essential tool in discovering the interactions between different levels of the system. The graphics program could also create movies of the cellular variables and could make graphs of any variable over time.

\section{Results}

\section{Formation of neuronal groups}

Although the synaptic efficacies of anatomical connections are difficult to determine in experimental preparations, they are straightforward to examine in this model network. Figure $6 \mathrm{~A}$ represents the $\mathrm{e} \rightarrow \mathrm{e}$ connection strengths of a representative $12 \times 6$ region of the network containing 144 excitatory cells and 2448 connections as they were initially assigned, prior to 


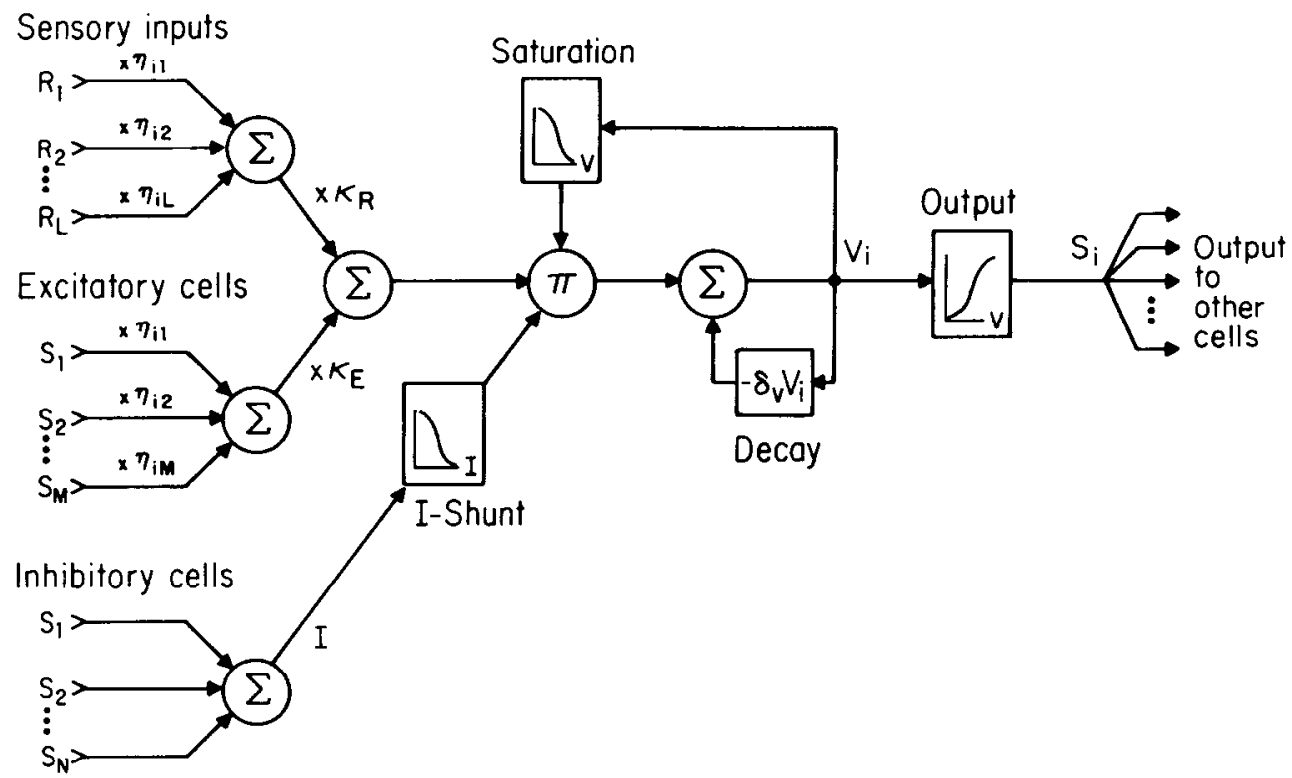

Figure 4. Schematic of the algorithm used to calculate the voltage, $v$, and the output, $s$, of a cell in the simulation (equations 1 and 2 ). The excitatory inputs from receptors $\left(\mathbf{R}_{\mathrm{i}}\right)$ and excitatory cells $\left(\mathrm{S}_{\mathrm{i}}\right)$ are multiplied by synaptic strength terms $\left(\eta_{i j}\right)$, summed separately, and are then multiplied by scaling factors $\left(\kappa_{E}, \kappa_{R}\right)$ and added together. This sum is multiplied by 2 fractions, one representing the shunting effect of the inhibitory input (I) and the other the reduction in synaptic current due to synaptic saturation (reduction of the transmembrane driving potential with increasing transmembrane potential). This product represents the net input to the cell. This input is then combined with a fraction $\left(1-\delta_{v}\right)$ of the voltage remaining from the previous time step. This yields the net voltage of the cell, which is then used to compute the output, $S$, of the cell by means of the sigmoidal function shown. The output is transmitted to other cells.

stimulation. The initial distribution was Gaussian (mean $=0.5$, $\mathrm{SD}=0.17$, range $=0.1-1)$. The initial $\mathrm{r} \rightarrow$ e connection strengths followed the same distribution. Note that, on average, the connection strengths appear similar across the network.

Figure $6 B$ shows neuronal groups that formed after stimulation of the hand; these groups are clusters of cells that have generally strengthened their mutual connections and weakened all others. The receptor sheet was stimulated in a random sequence of positions that eventually covered the entire hand. The stimulus was a $3 \times 3$ solid square that simultaneously activated a $3 \times 3$ square region of receptors, which in turn projected to a $9 \times 9$ region of the network. Each position on the hand was stimulated with this stimulus for 3 cycles of 6 steps on, 4 steps off. The stimulation was repeated until the entire hand had been covered 4 times. Within a given pass, the individual stimuli did not ovcrlap, but stimuli in each pass overlapped those from other passes with varying offsets, and they were applied in a different random order. The stimuli were applied without regard to the glabrous/dorsal border or to the individual finger and palm borders. Thus, stimuli straddled these borders to perhaps a greater degree than occurs naturally in the monkey.

During the first pass of stimulation, each stimulus excited an approximately $12 \times 12$ region of the network (due to the divergence of the input projection and the intrinsic connections). At the end of the first pass, there were relatively large clusterings of slightly strengthened connection strengths. With subsequent passes, the regions of activation shrank and became more intense as the groups became stronger, smaller, and more tightly packed. The group structure became relatively stable after about 3 passes, but the borders between groups continued to shift as the groups competed with each other for cells. During this com-
Table 2. Values of model parameters used in the simulation E I

\begin{tabular}{lccll}
\hline \multicolumn{2}{l}{ A. } & Neuronal & & \\
\hline$\theta_{s}$ & 20 & 20 & & output threshold \\
$\epsilon_{s}$ & 6 & 6 & & output nonlinearity \\
$\theta_{I}$ & 4 & - & shunt threshold \\
$\epsilon_{I}$ & 0.4 & - & shunt nonlinearity \\
$\theta_{v}$ & 80 & 80 & saturation threshold \\
$\epsilon_{v}$ & 10 & 10 & saturation nonlinearity \\
$\delta_{v}$ & 0.5 & 0.4 & voltage decay rate \\
$\kappa_{F}$ & 4.5 & 1 & c-cell input scalc factor \\
$\kappa_{R}$ & 1 & - & receptor input scale factor
\end{tabular}

B. Synaptic

\begin{tabular}{lll}
\hline$g$ & 0.1 & unmodified channel conductance \\
$g^{*}$ & 1 & modified channel conductance \\
$\kappa_{o p}$ & 0.4 & max. $\mathrm{O} \rightarrow \mathrm{P}$ transition rate \\
$\kappa_{o p}{ }^{*}$ & 0.4 & max. $\mathrm{O}^{*} \rightarrow \mathrm{P}^{*}$ transition rate \\
$\delta_{p o}$ & 0.05 & $\mathrm{P} \rightarrow \mathrm{O}$ decay rate \\
$\delta_{p o}^{*}$ & 0.05 & $\mathrm{P} * \rightarrow \mathrm{O}^{*}$ decay rate \\
$\kappa_{p}$ & 0.4 & max. $\mathrm{P} \rightarrow \mathrm{P}^{*}$ modification rate \\
$\delta_{p}$ & 0.01 & $\mathrm{P} * \rightarrow \mathrm{P}$ demodification rate \\
$\kappa_{m}$ & 0.3 & mod. substance growth rate \\
$\delta_{m}$ & 0.05 & mod. substance decay rate \\
$\theta_{o p}$ & 20 & $\mathrm{O} \rightarrow \mathrm{P}$ transition threshold \\
$\epsilon_{o p}$ & 2 & $\mathrm{O} \rightarrow \mathrm{P}$ transition nonlinearity \\
$\theta_{o p}{ }^{*}$ & 30 & $\mathrm{O} * \rightarrow \mathrm{P}^{*}$ transition threshold \\
$\epsilon_{o p}{ }^{*}$ & 3 & $\mathrm{O}^{*} \rightarrow \mathrm{P}^{*}$ transition nonlinearity
\end{tabular}

Values of both the neuronal and synaptic parameters used in the equations of the simulations. The $\mathrm{E}$ and $\mathrm{I}$ columns $(A)$ are the e- and i-cell parameters, respectively; the dashes in the I column refer to parameters which are irrelevant because of the particular connectivity used, i.e., there were no $\mathrm{i} \rightarrow \mathrm{i}\left(\theta_{l}, \epsilon_{l}\right)$ or $\mathrm{r} \rightarrow \mathrm{i}\left(\kappa_{R}\right)$ connections. The various $\theta$ 's and $\epsilon$ 's are parameters that determine the inflection point and the slope at the inflection point, respectively, of the sigmoidal functions (see equation 1) used to simulate the nonlinear kinetics of state transitions. 

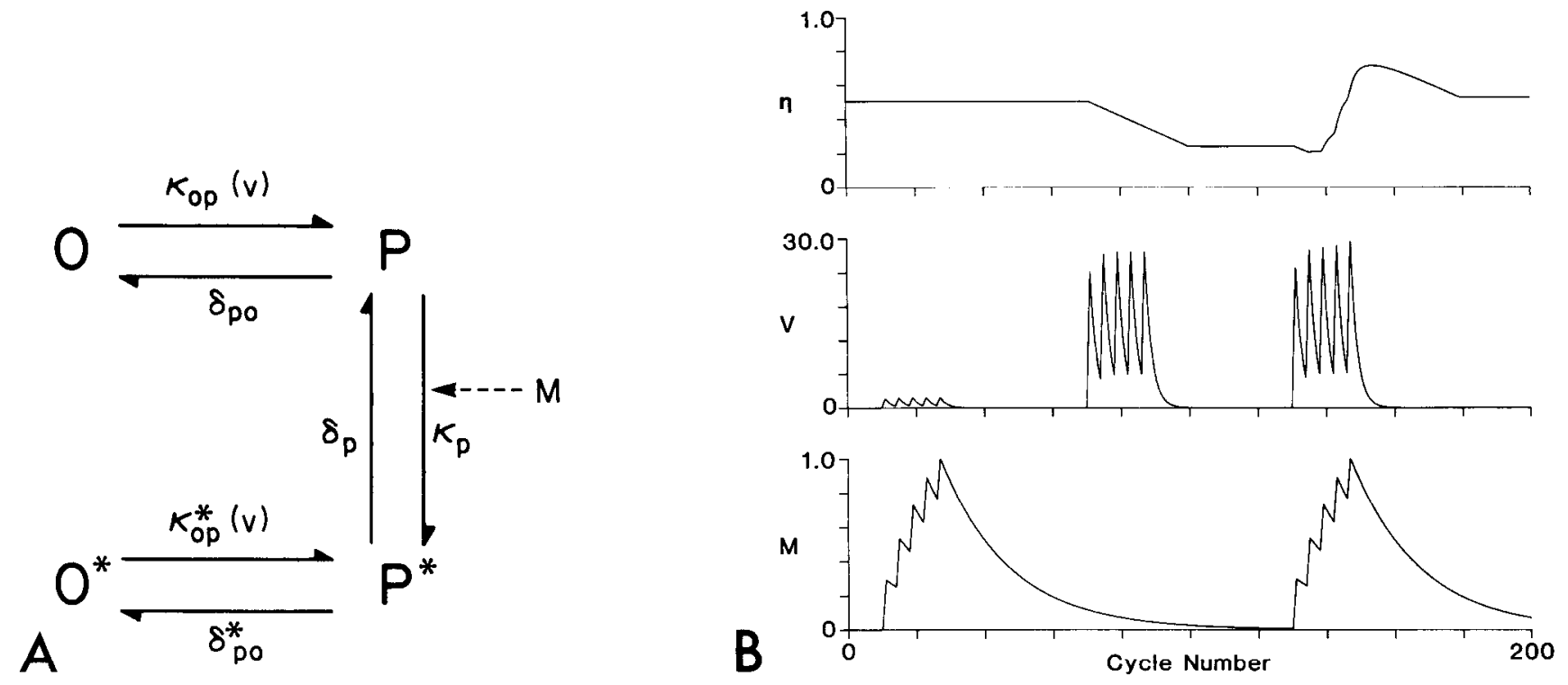

Figure 5. The synaptic modification rule. $A$, Transition scheme governing postsynaptic plasticity in the model. $\mathrm{O}, \mathrm{P}, \mathrm{O}^{*}$, and $\mathrm{P}^{*}$ represent the fraction of channels at a given synapse that are in each of the 4 conducting channel states. $\kappa(v)$ and $\kappa^{*}(v)$ are the state transition rates and are increasing sigmoidal functions of cell voltage, $v ; M$ is the amount of modifying substance, and the remaining Greek letters are constants given in Table 2B. The modified states $\mathrm{O}^{*}$ and $\mathrm{P}^{*}$ have a larger conductance than the unmodified states $\mathrm{O}$ and $\mathrm{P}$. Thus, synaptic strength is increased in the M-dependent $\mathrm{P} \rightarrow \mathrm{P}^{*}$ transition, and weakened by decay from $\mathrm{P}^{*} \rightarrow \mathrm{P}$. The ratio of $\mathrm{P}$ channels to $\mathrm{O}$ channels is determined by the voltage history of the postsynaptic cell. $B$, Illustrative simulation of the postsynaptic rule in a simple network. The network consists of one postsynaptic cell receiving multiple inputs; changes in the strength $(\eta)$ of a single given synapse are related to the modifying substance $(M)$ present at the synapse and the voltage $(V)$ of the postsynaptic cell. At the start, $\eta=0.5$, with $4 / 9$ of the channels in state $O^{*}$ and $5 / 9$ in state $O$. During the first set of 30 cycles, only the given synapse was stimulated with 5 short bursts. The $V$ produced was insufficient to induce channel transitions into the $P$ or $P^{*}$ states, and $\eta$ therefore remaincd unchanged despite the fact that the stimulation produced significant amounts of $M$. During the second set of 30 cycles, a large number of other synapses were stimulated and the large heterosynaptically generated $V$ drove the $\mathrm{O}_{\text {and }} \mathrm{O}^{*}$ channels into the $\mathrm{P}$ and $\mathrm{P}^{*}$ states, respectively. Because the given synapse was not stimulated, $M$ was low, and the predominant transition was the $\mathrm{P}^{*} \rightarrow \mathrm{P}$ decay that reduced $\eta$. The third set of 30 cycles was the same as the second, but the given synapse was also stimulated, producing high concentrations of $M$. In this case, the predominant transition was $\mathbf{P} \rightarrow \mathrm{P}^{*}$ and the synapse was strengthened.

petition, the sizes and shapes of the groups changed, but in accord with the notion of group confinement (Edelman and Finkel, 1984), there was a minimum and maximum allowable size.

Group formation was fairly robust with respect to the stimulation protocol. Protocols employing different random sequences of stimulation, or using stimuli that varied over a several-fold range of sizes, intensities, or durations all led to generically similar group structures. Neither the sizes nor the locations of the groups were determined solely by the character of such input stimulation. Each receptor projects to a $7 \times 7$ region of the network, and each group occupies a small arbitrary (roughly $3 \times 3$ ) domain within the $12 \times 12$ region initially excited by the stimulus. Furthermore, all network areas were stimulated equally. Thus, the locations of the groups were not prespecified by the anatomy or the input but depended upon local inhomogeneities in the patterns of connections, their initial strengths, and the historical sequence of stimulation.

Figure $6 C$ is a magnified $(\times 2.5)$ view of the $12 \times 6$ region in Figure $6 B$ that contains the groups marked " $*$ " and " $\dagger$ ". It is evident that most cells belong to only one group, although a few cells have strong connections to 2 groups (see the green connections of group $*$ ). With further stimulation, these cells are usually captured exclusively by one group. As a result of group

\footnotetext{
Figure 6. The formation of neuronal groups. $A$, Initial $\mathrm{e} \rightarrow \mathrm{e}$ connection strengths $(\eta)$ before stimulation of a small $(12 \times 6)$ region of the network chosen for illustration and containing 144 excitatory cells and 2448 connections. A straight line has been drawn between all pairs of connected cells (the lines do not indicate the directionality of the connections), and the locations of the cells are marked with circles. The color of each line represents the strength of that connection, as indicated by the scale at the bottom. To reduce superposition of lines, the inputs and outputs are drawn to points slightly above and below the cells, respectively. About $16 \%$ of the connections are between the same pairs of cells and are represented by single lines. $B$, The $\mathrm{e} \rightarrow \mathrm{e}$ connection strengths of the network following external stimulation of the entire surface of the hand with small locally correlated stimuli in a random sequence of positions. Neuronal groups have formed due to clusters of nearby cells strengthening their mutual connections while weakening most others. These groups collectively determine the receptive field properties of their component cells and organizc the receptive field map via group competition. The 2 adjacent groups marked "*" and " $\dagger$ " are located within the region shown in $A$ and $C$. $C$, Magnified ( $\times 2.5)$ view of the $12 \times 6$ region of $B$ containing the groups marked "**" and " $\dagger$ " (same region as in $A$ but after group formation). Note the clear blue borders (composed of many weakened connections) separating the groups. The border of weakened connections around a group forms a "moat," which protects it from encroachment by other groups and is a stabilizing force in the network. Borders between groups shift as cells are captured by competing groups. The properties of these group borders are responsible for the dynamics of functional boundaries between hand regions as seen in the global receptive field maps (see Fig. 9). In the particular case shown here, the group */ $\dagger$ structural border corresponds to a glabrous/dorsal functional boundary (see Fig. 9B).
} 
B

C
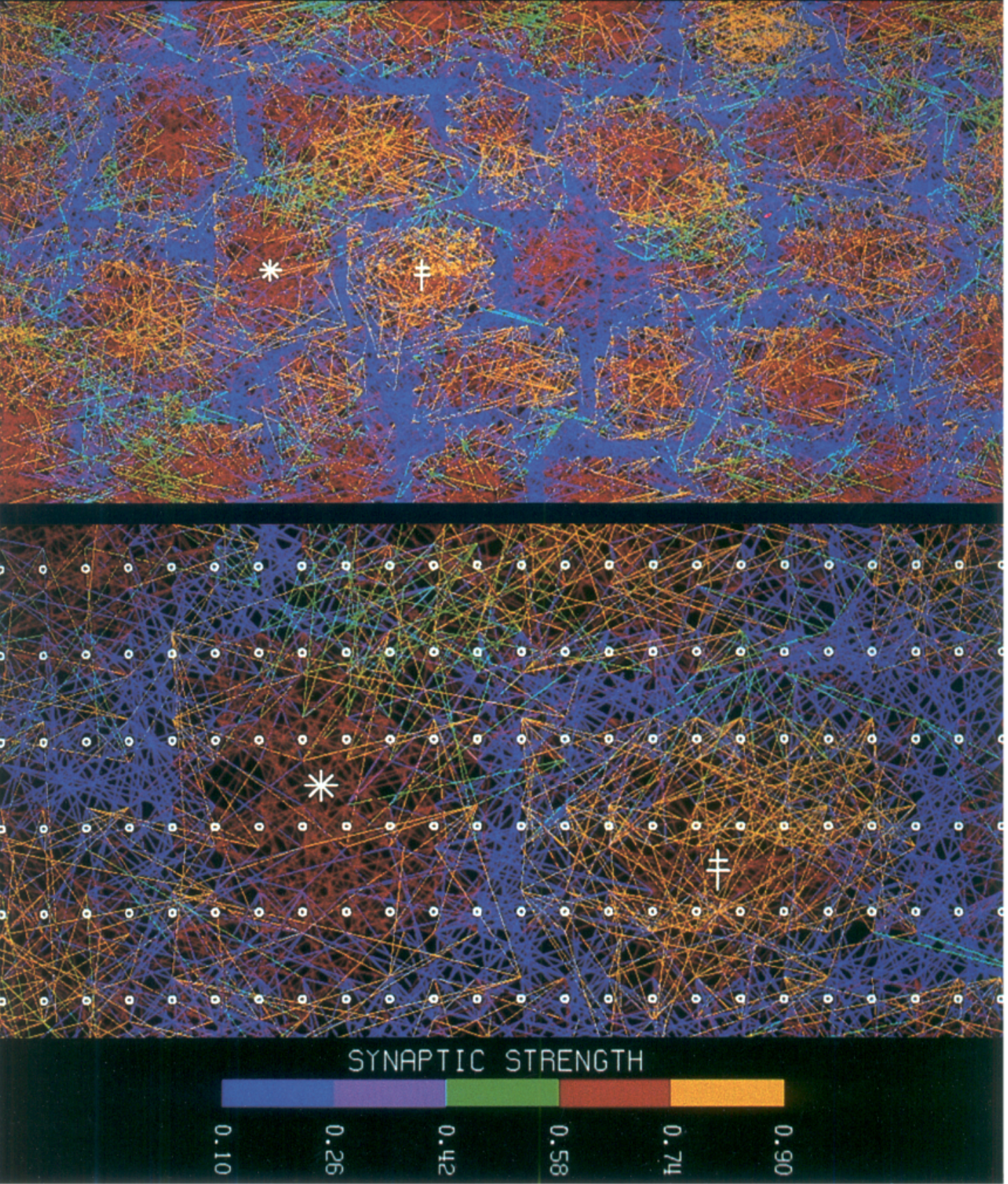
Figure 7. Receptive field plots of representative cells from the adjacent groups labeled ${ }^{*}$ and $\dagger$ in Figure $6 B$. The receptive fields of group * are shown on the left and those of group $\dagger$ on the right, both before (top) and after (bottom) the groups had formed. The average response of the cell to stimulation at cach point of the hand is represented by a block, the shade of which codes the strength of the response (as indicated by the scale at the bottom of the fig.) and the size of which is equal to the size of the stimulus used. Each finger contains 8 receptors widthwise and 12 receptors lengthwise, thus the rectangular receptive field in the bottomleft panel covers an area of $3 \times 3$ receptors. Small dots mark the locations of the centers of the glabrous and dorsal regions of the hand that project to the cell. The $\mathrm{X}$ dimension of the hand shown here is compressed by a factor of 2 in order to facilitate display. (This accounts for the rectilinear appearance of the actually square-shaped receptive fields). There are 3 important changes in the receptive fields as a result of group formation: (1) The peak response has increased and shifted; (2) the size of the receptive field has decreased; and (3) the receptive fields have become exclusively glabrous or dorsal.
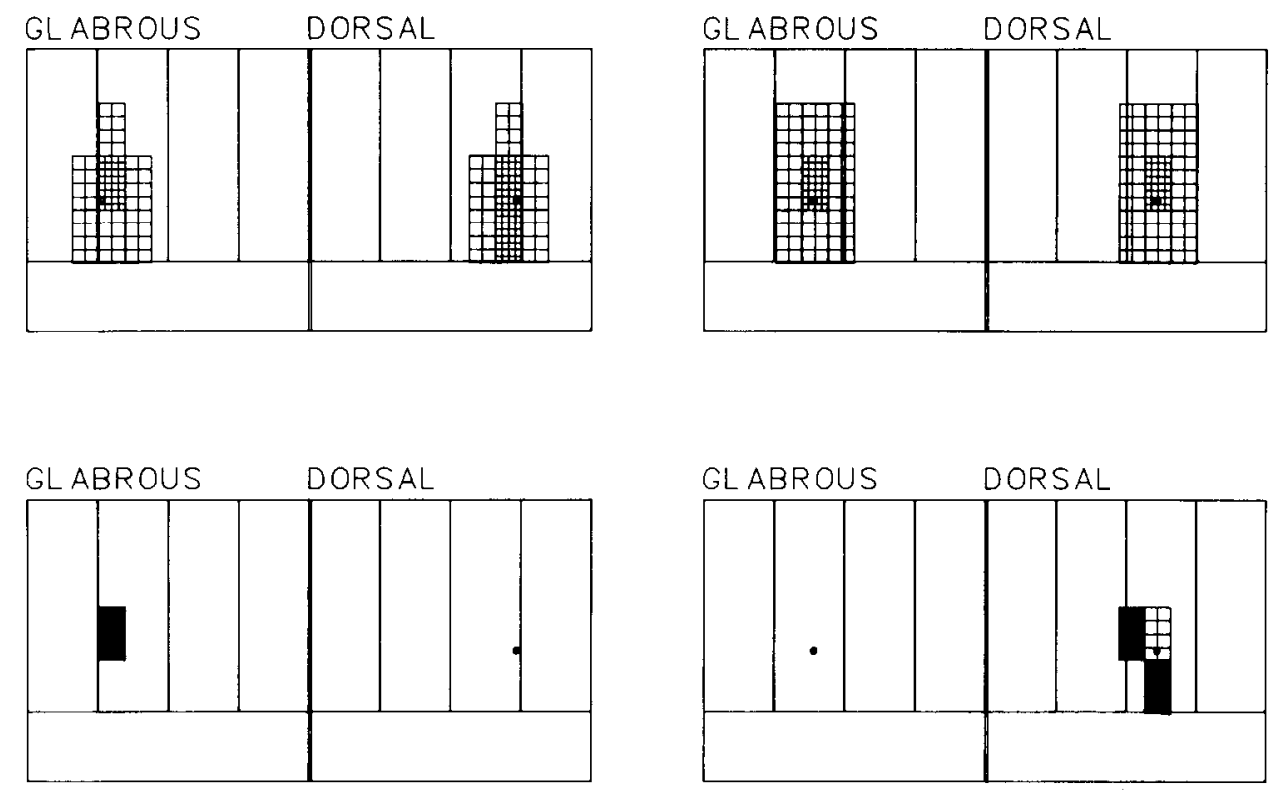

formation, the distribution of connection strengths changes from a Gaussian with a mean of 0.5 to a bimodal distribution with widely separated peaks. This bimodal distribution is stable once the groups have formed, even though the groups continuc to shift, grow, and shrink as they compete with each other.

\section{Formation of an ordered topographic map}

The formation of groups organized the receptive fields of cells in the network. This can be seen by considering the 2 adjacent groups labeled $*$ and $\dagger$ in Figure $6, B$ and $C$. Figure 7 shows the receptive fields (see Materials and Methods) of a typical cell in each of these 2 regions before and after the groups had formed. The receptive fields of cells in the initial network were large, occupying the major part of one finger, they were relatively weak, and all cells had receptive fields on both the glabrous and the dorsal surfaces of the hand. This is as would be expected given the anatomy: each cell receives an equal number of inputs from the glabrous and dorsal surfaces. With the formation of neuronal groups, however, 4 dramatic changes are seen in the rcceptive fields of the component cells: (1) the sizes of the receptive fields decrease dramatically, (2) the overlap of the fields of cells within a group increases, (3) the strengths of the responses increase, and (4) the receptive fields of cells become either exclusively glabrous or exclusively dorsal, but not mixed. The case of cells with receptive fields centered on the lateral or medial surfaces of the fingers or palm is, of course, an exception inasmuch as these fields can extend onto both surfaces of the hand. Some receptive fields straddle the borders between digits as a result of the stimulation protocol which did not respect these borders (see Materials and Methods). Such receptive fields are, however, occasionally observed in the monkey (Merzenich et al., 1983a).

The local topography of the mapping is shown in Figure 8, which is a display of the centers of the receptivc ficlds of cells encountered in 2 linear tracks across the network (corresponding to 2 tangential electrode penetrations in cortex). One track was made horizontally across the network (at a midvertical position) and encountered 64 cells from left to right. The receptive field centers of these cells are marked with rectangles. The second track was made vertically from the top to the bottom of the network, and the receptive field centers of the 32 cells encountered are marked with circles. The receptive field centers were determined by weighting the contribution of each receptor in the receptive field by its response-thus these are not strictly the geometrical centers, but the centers of activity of the receptive fields. Figure 8 (top) shows the receptive field centers before group formation (corresponding to the network in Fig. 6A). The initial topography is such that the receptive field centers move in a generally smooth progression across the surface of the receptor sheets. The small degree of scatter arises from the random component in the underlying anatomical connectivity (see Materials and Methods). Note that all cells initially have both glabrous and dorsal components to their receptive fields. Figure 8 (bottom) shows the receptive fields of the same cells determined after the formation of neuronal groups (corresponding to Fig. $6 B$ ). The receptive field centers are numbered according to the order in which cells were encountered in the 2 tracks. (Primed numbers refer to the vertical track; unprimed numbers refer to the horizontal track.) The receptive field centers have shifted into a number of clusters separated by gaps. The clusters cor- 

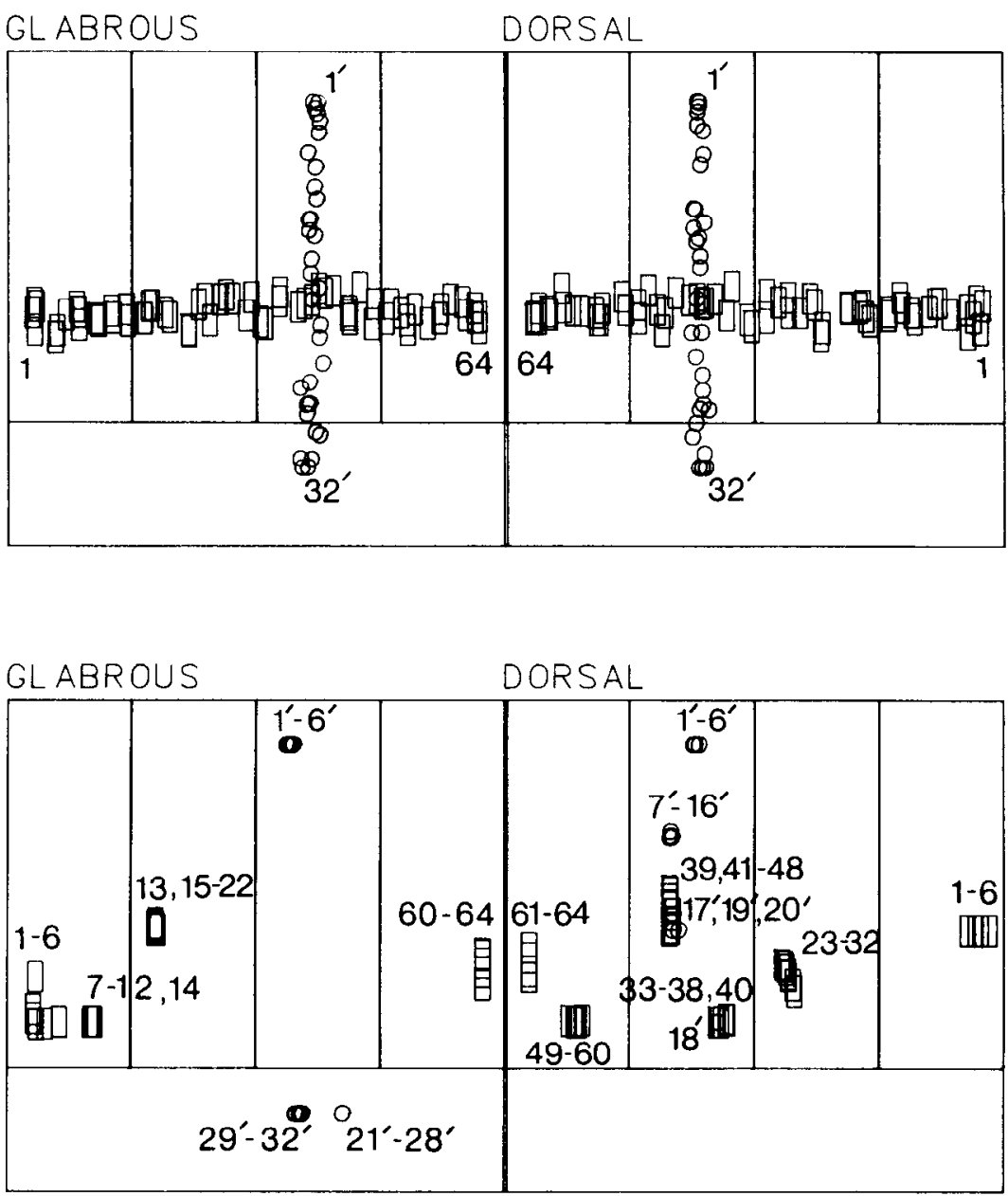

Figure 8. Local topography of the mapping. Top, Before group formation. Rectangles mark the centers of the receptive fields of 64 cells encountered as the network was traversed from left to right along a horizontal line (located midway between top and bottom of the network in the vertical dimension). Circles mark receptive field centers of 32 cells found along a second track running vertically from top to bottom of the network. The first and last receptive field centers in each track are numbered (those in the vertical track are primed, e.g., $1^{\prime}$ and $32^{\prime}$ ). Prior to group formation, each cell has both glabrous and dorsal receptive field components, and the receptive field centers move in a generally smooth linear progression across the receptor surface. Note that the rectangles correspond to the size of a single receptor. Bottom, After group formation. Receptive field centers of the same cells as in Top, labeled in the order of encounter along the same horizontal (rectangles) and vertical (circles) tracks. Each track passes through several neuronal groups (see Fig. $6 B$ ). Cells in the same group have superposed or highly overlapped receptive field centers. But the receptive field center shifts considerably between adjacent cells belonging to 2 different groups (e.g., cells 48 and 49). The transition between cells belonging to glabrous and dorsal groups is also seen (e.g., cells 22 and 23 , or cells $20^{\prime}$ and $21^{\prime}$ ). Note that the horizontal and vcrtical tracks intersect, accounting for the superposition of some receptive field centers in the 2 tracks. respond to the receptive fields of cells in the same group; as different groups are encountered along the track, the receptive fields locations shift. Thus, in the horizontal track, cells 1-6 were at the network edge and had mixed receptive fields, cells $7-12$ and 14 were in a glabrous group, cells 13 and 15-22 were in another glabrous group, cells 23-32 were in a dorsal group, 33-38 and 40 in another dorsal group, and so on. Cells in the same group share highly overlapped receptive fields, and as one passes from one group to the next, the receptive field location suddenly shifts, even between adjacent cells. Note that the appearance that large regions of the receptor sheet are not covered follows from the fact that only the centers of the receptive fields are displayed, and again only those from a single vertical or horizontal row of cells.

Receptive field changes following group formation are seen best in the receptive field map. This map (Fig. 9) displays 2 types of receptive field information: for each excitatory cell in the network, the map indicates (1) the digit or palm on which the center of the receptive field is located and (2) whether the receptive field is on the glabrous surface, dorsal surface, or both. The map is coded such that receptive field locations on adjacent digits or palmar regions are distinguished by alternate light-gray and dark-gray solid fill background. In addition, cells with receptive fields on the glabrous surface are individually marked with a small black rectangle, those with receptive fields on the dorsal surface are marked with a small white rectangle, and cells with mixed glabrous and dorsal responses are unmarked. A cell is labeled "glabrous" if $80 \%$ or more of its summed response to all stimuli is to glabrous stimuli; conversely, it is labeled "dorsal" if more than $80 \%$ of its summed response to all stimuli is to dorsal stimuli. If a cell meets neither of these conditions, it is labeled "mixed." The initial receptive field map (Fig. 9A) shows that topographic organization is present before groups have formed; this follows from the topography inherent in the anatomical projection from the hand to the network. Initially, all cells respond to both dorsal and glabrous inputs (i.e., have "mixed" receptive fields). Although the exact balance varies, the initial difference between dorsal and glabrous influences was equal to within $20 \%$ for all cells.

After groups had formed, a well-ordered topographic representation of the fingers and palm was observed, with segregated zones of representation of the dorsal and glabrous surfaces (Fig. $9 B$ ). Although different stimulation schemes yielded different receptive field maps, all schemes employing locally correlated stimuli on the hand resulted in ordered maps with compact regions of dorsal and glabrous representation. Within each group, the nonlinear and highly cooperative interactions between voltage and synaptic strengths lead to similar changes in all cells of the group in response to glabrous versus dorsal stimulation. The receptive field choice depends upon the early balance of glabrous versus dorsal stimulation received by the group and by nearby groups. Early biases are amplified into the resulting organiza- 

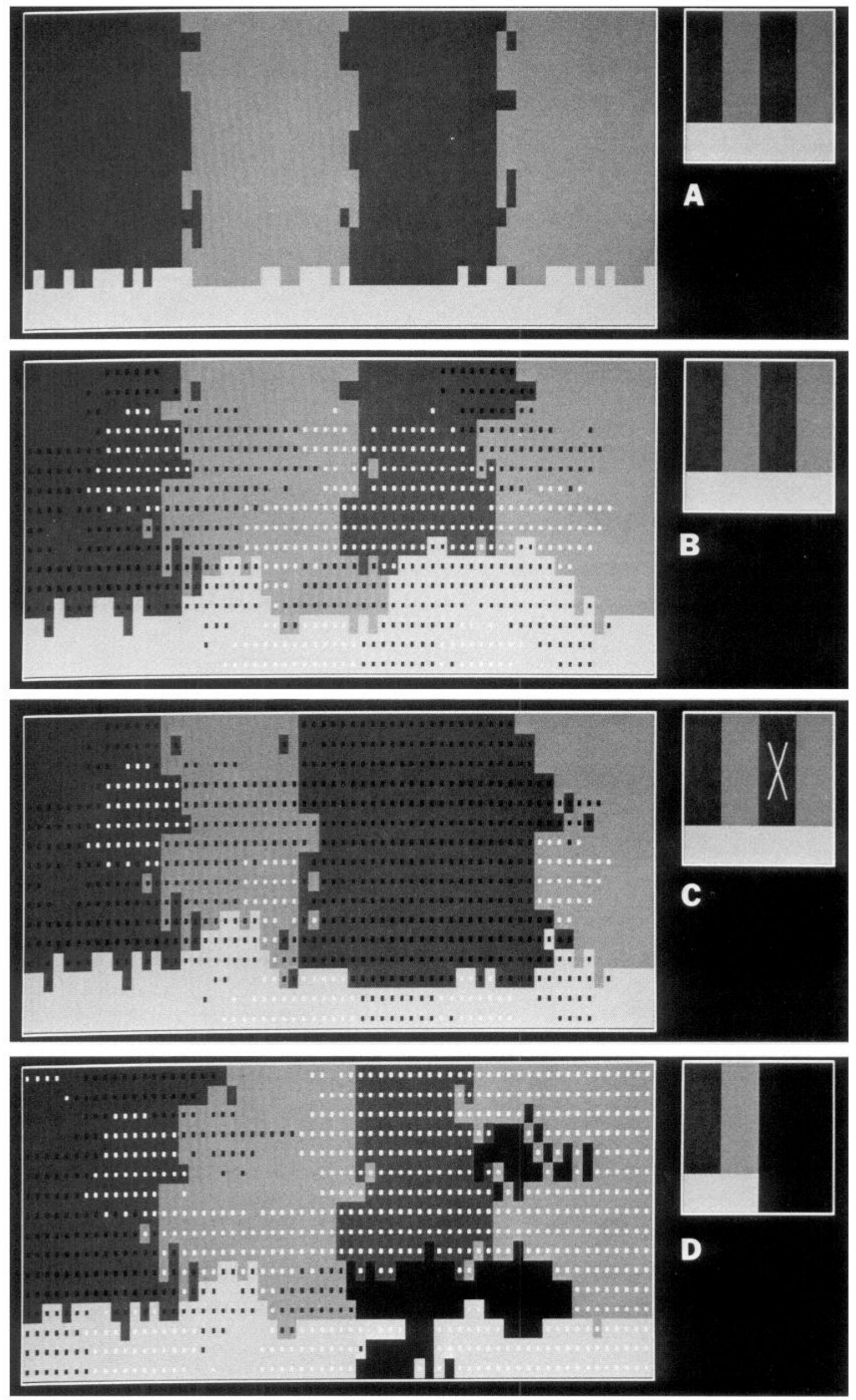
tion. The ratio of groups responding to glabrous versus those responding to dorsal stimulation depended rather sharply (see Discussion) upon the relative balance of stimulation to the 2 surfaces of the hand. In the example shown here, the glabrous and dorsal surfaces were stimulated equally, and of the 19 nonmixed groups present, 12 are glabrous and 7 are dorsal (the dorsal groups contain slightly more cells on the average; thus, $59 \%$ of the nonmixed cells are glabrous and $41 \%$ are dorsal). Although this distribution appears skewed in favor of glabrous groups, the asymmetry is not statistically significant and follows from the small total number of groups. Nonetheless, the apparent tendency of the glabrous and dorsal groups to form in domains exclusive of the other (see Fig. 9B) suggests that whether a group becomes a glabrous or dorsal responder may depend to some degree upon the nature of nearby groups.

In this simulation, $32.3 \%$ of the e-cells remained mixed, i.e., their responses to dorsal or glabrous stimulation were equal to within a factor of 4 . Most of these cells were found at the borders of the network; $95 \%$ of the cells within 3 boxes of the righthand border were mixed, $51.2 \%$ of the cells within 3 boxes of the other borders were mixed, while only $6.3 \%$ of the nonborder cells (located within the central $26 \times 10$ region of the network) were mixed. The high proportion of mixed cells near the borders is due to the higher connection density at these sites (see Materials and Methods). Higher local connection densities generate higher cellular voltages in response to local stimuli, and so can be more easily strengthened from a weakened state, thus preventing either the dorsal or glabrous stimuli from gaining domination of the group.

The very high percentage of mixed cells at the right-hand border is due to the fact that the glabrous/dorsal surfaces are continuous along the middle of the input array (which projects to the right-hand edge of the network). Thus, during group formation, stimuli straddle this midline and glabrous and dorsal receptors are coactivated. This naturally occurs at the edges of the monkey hand (Merzenich et al., 1978).

The mixed receptive fields of cells in the central region of the network are due to groups competing for cells. An examination of the intrinsic connection strengths of the network revealed that most $(88 \%)$ of these mixed cells were located between groups with opposite receptive field types and that they shared strong connections with both groups. As the network receives additional stimulation, these mixed cells are generally captured by one group or the other and then exhihit the receptive field type of that group. Because group competition is an ongoing process in the network, we expect that there will always be a small percentage of mixed cells in the central region, located predominantly between groups of opposite receptive field type.

\section{Simulations of reported experiments}

We used the network with the group structure shown in Figure $6 B$ and the receptive field map shown in Figure $9 B$ to simulate a number of reported experiments (Merzenich et al., 1983a, b, 1984; Jenkins et al., 1984). We will discuss 2 such simulations that yielded results qualitatively similar to those observed experimentally.

\section{Results of simulated repetitive tapping of digit 2}

The first simulation involved increased stimulation of a local region of the hand and was designed to investigate factors that control the magnification factor (the ratio of the area of a cortical representation to the area of the corresponding body region represented). We applied repeated stimulation to the glabrous surface of digit 2; the same protocol was employed as in group formation, but the individual stimuli were 4 times as intense $\left(\kappa_{R}=4\right)$. Digit 2 was stimulated exclusively, and its entire surface was covered several times. This stimulation was applied to the network that already had the mature group structure shown in Figure 6, $B$ and $C$.

The stimulation produced the new receptive field map shown in Figure $9 C$. The area of representation of the glabrous surface of digit 2 expanded greatly (here by a factor of 13.7 ) to nearly the anatomical limit of the underlying projection. The total area of representation of digit 2 (both glabrous and dorsal surfaces) increased by a factor of 2.3. The borders of the representation of this digit expanded in all directions, increasing the magnification factor of the digit at the expense of that of neighboring regions. The representations of digits 3 and 4 were relatively unchanged.

The extent to which map borders shift depends upon the intensity of the stimulation, provided that the intensity exceeds certain minimal levels. However, in general, the more intense the stimulation, the greater is the change in group structure. In the example shown here, the group structure was radically affected with many groups merging due to the intense, locally correlated nature of the stimuli. Less intense stimulation leaves

\footnotetext{
$\leftarrow$

Figure 9. Receptive field maps after normal stimulation and perturbations. The position of each excitatory cell in the network (the 2 excitatory cells at the same network grid point are displayed in adjacent positions) is marked with a block colored according to the location of the center of the cell's receptive field on the hand, as indicated by the diagram of the hand (which is compressed along the X dimension by a factor of 2 to facilitate display). Cells that responded primarily to stimulation of the glabrous or dorsal surface are marked with a small black or white rectangle, respectively, while cells with mixed glabrous/dorsal responses are not marked. $A$, Prior to group formation, topographic order is present (inasmuch as the projection from the hand is topographic by design), but the receptive fields of all the cells are mixed, reflecting the fact that initially the inputs from both surfaces are roughly equal. $B$, After group formation, the map borders representing hand regions have shifted because of the establishment of group borders, and most of the cells are exclusively glabrous or dorsal responders. A significant fraction of the cells retain mixed receptive fields, but these lie primarily on the edges of the network (see text). $C$, Starting with a network with well-formed groups (Figs. $6 B, 9 B$ ), glabrous digit 2 was repetitively stimulated (as indicated by the $\mathrm{X}$ on this digit in the hand diagram). Note that the representation of glabrous digit 2 has expanded greatly (here by a factor of 13.7 ) at the expense of the representations of adjacent digits and of its own dorsal representation. This expansion is due to the growth of the groups representing glabrous digit 2, as well as to other groups having switched their receptive fields. Further stimulation does not produce more expansion because of the anatomical limits of the projection. $D$. Starting with the same network with wellformed groups (Figs. $6 B, 9 B$ ), the inputs from glabrous digits 1 and 2 and subjacent palm (as indicated by the black regions in the hand diagram) were interrupted, corresponding to a transection of the median nerve in monkeys. All other extrinsic connections remain intact, including all connections from the dorsal surface. Cells unresponsive to all stimulation are colored black. There is a new representation of the dorsum of digits 1 and 2 and subjacent palm in the network region, which, before the transection, contained a representation of the glabrous surfaces of these regions. The new representation is not complete, and there remain silent, unresponsive regions. These regions remain silent unless very intense stimulation is used. This results from the fact that their connections with the dorsum are so weak that normal stimulation docs not produce voltages high enough to enable synaptic strengthening.
} 
the group structure unchanged, but such stimulation gives rise at maximum to only a 2 -fold increase in the magnification factor. Even after intense stimulation, the original group structure can be largely recovered by applying several passes of normal stimulation to the hand, and this additional stimulation leaves the increased magnification factor of digit 2 intact.

The results of this simulation correspond reasonably closely to the experimental result found after repeated tapping on the glabrous surface of a single digit in the owl monkey (Jenkins et al., 1984). The experimentally reported observation was made after hundreds of thousands of repeated stimuli; those in the simulation occurred after only hundreds. There is no reported evidence on experimental changes after shorter stimulation protocols, but it is likely that the simulated connections are relatively more "plastic" than those in the real nervous system -a compromise that is necessary for compressing the simulation into a manageable number of iterations.

\section{Results of simulated nerve transection}

The second simulated experiment involved decreasing the net stimulation to a region. This was accomplished by transecting the connections from half of the glabrous surface of the hand (digits 1 and 2 and subjacent palm), corresponding roughly to transection of the median nerve in the monkey. Once cut, there was absolutely no activation from the median half of the glabrous hand to the network. The receptors from the rest of the hand remained intact, and the entire hand (both affected and unaffected regions) was lightly stimulated ( 3 steps on, 7 steps off) in a random sequence with a $3 \times 3$ stimulus. This background stimulation was to simulate the light touches that an animal would unavoidably encounter.

We examined the receptive field maps at various times after the transection. Immediately after the cut, i.e., before any stimulation of the hand, the cells that formerly had receptive fields in the denervated region were no longer responsive. These "silenced cells" received weak conncetions from the dorsal surface of the transected region, and small postsynaptic potentials were observable. This population of weak inputs, acting together, produced sufficient postsynaptic voltage to strengthen their connections over time due to the voltage-dependent properties of the synaptic rule. This ability to strengthen such weakened connections is a property that distinguishes voltage-dependent (Finkel and Edelman, 1987) from output-dependent synaptic rules such as the Hebb rule (Hebb, 1949).

Shortly after the simulated transection (i.e, after 2 passes of stimulation), the former area of representation of the median half of the glabrous surface is occupied by a representation of the corresponding dorsal surface of the hand (Fig. 9D). This new representation is topographic, but the borders between the fingers and with the palm are different than before. There is a large silent region (the blackened cells) in which cells are still not responsive. Note that the representation of the unaffected glabrous half of the hand is relatively unchanged from that seen before the transection. Note also that the locations of the borders and the magnification factors of digits 1 and 2 and the palm differ from the original glabrous representation, yet the map shows continuity and compactness. The group structure corresponding to this map (not shown) was almost identical to the pretransection structure.

With normal stimulation, the silent regions remain essentially constant despite extended periods of stimulation (12 passes). With repeated stimulation, the silent areas eventually become responsive to dorsal stimuli, providing that the intensity of the stimulation is increased. As discussed above, such an increase also changes the group structure. These results are very similar to those found experimentally with the recovery from transection of the median nerve in owl monkeys (for comparison, see Figure 2 in Merzenich et al., 1983b).

\section{Discussion}

The major results of the computer simulations reported here concern the dynamic control of topographic map organization as a result of the formation of neuronal groups. We have shown that a network with locally connected excitatory and inhibitory cells, divergent overlapping extrinsic connections similar to those of the thalamocortical projection (Gilbert and Wiesel, 1979; Landry and Deschenes, 1981), and realistic synaptic plasticity rules will spontaneously organize into neuronal groups when activated by locally correlated stimulation of the input array. After group formation, the receptive fields of cells in the network become smaller, stronger, and exclusively restricted to either the glabrous or dorsal surface of the hand, despite the fact that all cells of the network still receive equal numbers of anatomical connections from both surfaces of the hand. Groups act collectively to segregate the network into exclusively glabrous or dorsal domains in which all cells have similar receptive fields. After repetitive tapping of a local region, or transection of extrinsic fibers, there are en masse shifts in the receptive fields of cells in the affected groups, leading to dramatic changes in the receptive field map of the network. The results of these simulations correspond closely to results seen in monkey cortex after similar perturbations.

Group formation depends upon the positive feedback between changes in connection strength and cellular potential. The properties of the synaptic rule are such that the same conditions that strengthen the active synapses onto a cell weaken the inactive ones. In general, this prevents a cell from belonging to more than one group and ensures that the borders between groups remain sharp even though their locations may shift. Groups form under a variety of stimulation protocols; however, all such protocols lead to a point of stability after which further stimulation does not significantly change the group structure.

The segregation of the map into neuronal groups is similar to the tendency of all systems with short-range excitation and long-range inhibition to organize into domains (Turing, 1952; Meinhardt, 1982). The critical difference is that neuronal groups, once formed from the fixed anatomy, act as independent competitive organizers of a functional map out of that divergent and overlapping anatomical substrate. The receptive field of each group is restricted to either the glabrous or dorsal surface depending upon historical events: whether the group received greater stimulation from one side or the other and whether one side initially dominated the stimulation to the group and established a slight advantage. Such competitive advantages tend to become amplified during selection, leading to the dramatic strengthening of one class of inputs and the weakening of others.

It is important to note that group formation involves synaptic modifications in both intrinsic and extrinsic connections. It is not known in vivo, whether these changes are independent or whether one necessarily follows upon the other. However, our experience with various trial simulations suggests that group formation is most robust if intrinsic connections are plastic concurrently with extrinsic connections. The scheme yielding the best defined and most densely packed groups was one in 
which the synaptic plasticity parameters for extrinsic and intrinsic connections were identical (Table 2), and in which the voltage necessary to weaken connections was higher than that required to strengthen them $\left(\theta_{o p}{ }^{*}>\theta_{o p}\right)$. In this scheme, the voltage required to weaken extrinsic connections can be achieved only in a well-formed group (due to the stronger intragroup intrinsic connections and therefore larger voltages within a group); thus, only the cells in a group can refine their receptive fields by weakening some subset of the extrinsic connections they receive. All subsequent results were found to depend on the quality of the groups formed. In networks with poor group structure, the cells had large receptive fields that retained components on both surfaces of the hand.

One of the most critical features of the present computer model is the robustness of the results with respect to changes in the parameters. Of the 23 neuronal and synaptic parameters in the model (Tables 1 and 2), we found the most critical relationships to be (1) the number of inhibitory inputs to a cell relative to the threshold of the inhibitory shunt $\left(\theta_{l}\right) ;(2)$ the scale factors of the excitatory inputs $\left(\kappa_{E}, \kappa_{R}\right)$ relative to the firing threshold $\left(\theta_{s}\right)$; (3) the voltage threshold for channel transitions $\left(\theta_{o p}, \theta_{o p}{ }^{*}\right)$ with respect to firing threshold $\left(\theta_{s}\right)$; and (4) the ratio of the rates for modification and demodification $\left(\kappa_{p}, \delta_{p}\right.$, respectively).

Although the results were largely independent of the values of the neuronal parameters chosen, they obviously required selfconsistency (e.g., synaptic saturation must occur at a voltage greater than the firing threshold). Of somewhat greater sensitivity were the parameters involved in the synaptic plasticity rules. By adjusting the synaptic plasticity parameters (Table 2), we could create groups of different sizes or different packing densities, groups surrounded by regions of weakened connections of various widths, and groups with wider or narrower receptive fields.

The critical feature of the postsynaptic rule in group formation and map organization is the ability to strengthen or weaken subsets of connections in a context-dependent fashion (i.e., based on heterosynaptic input voltages). Although channel- and receptor-based modification schemes seem ideally suited to such heterosynaptic mechanisms, a variety of other synaptic mechanisms might subserve similar roles (Finkel and Edelman, 1987). We have not considered presynaptic modifications in the present simulations; however, previous studies (Finkel and Edelman, 1985) have shown that pre- and postsynaptic mechanisms operating in parallel lead to a wide class of properties that can, for example, couple short- and long-term modifications.

The model tested here was deliberately constructed to be a minimal one, and it is not surprising that its performance left a number of problems to be solved. The most serious shortcoming is that the model does not follow the inverse rule (Edelman and Finkel, 1984), namely, that receptive field size is inversely related to magnification factor (Daniel and Whitteridge, 1961; Sur et al., 1980). In particular, the large increase in magnification factor in the "repetitive tap" simulation was not accompanied by receptive field decreases on glabrous digit 2 . One reason for this failure is that, after group formation, many receptive fields are as small as possible, and are unable to shrink further. Another possible reason is that, due to limitations on network size, each peripheral region (e.g., each finger) is represented by just a few groups, too few presumably to generate the intergroup interactions responsible for the inverse rule.

There are also several remaining difficulties related to the properties of the mapping. In the simulations shown here, $7 \%$ of the surface of the hand was not represented in the network. These nonrepresented receptors mapped to cells located at the extreme borders of the network. It is not clear from reported maps (Merzenich et al., 1978) whether the complete surface of the hand is actually represented in areas $3 b$ or 1 , and it might be of particular interest to examine the completeness of representations located near cytoarchitectonic borders. Another residual problem is that biasing the ratio of glabrous to dorsal stimulation by a factor of 2 results in a map in which all but a few groups primarily respond to the more frequently stimulated surface. This appears to be an unusually sharp dependence on the stimulation ratio of glabrous to dorsal inputs. The cause of this problem is related to the relatively small number of groups in the network, the rate of group formation, and the stereotyped nature of our stimulation. Finally, it was observed that under certain relatively rare circumstances, bursts of activation of moderate intensity were not self-extinguishing. This problem could have been eliminated by introducing synaptic depression or long-lasting hyperpolarizing inhibition, but hecause the pathology was rare, we decided to simply set the voltages of all cells to zero between successive stimulations.

The functional somatosensory mapping problem we have considered contrasts in some respects to that confronting the primary visual cortex. The mechanisms underlying the segregation may be analogous in both cases, and may result from a large degree of local coactivation; however, the functional properties of the mappings appear to differ. In the visual system, corresponding points on the 2 retinae are frequently, if not usually, costimulated. In the somatosensory cortex, corresponding points on the 2 surfaces of the hand are rarely, if ever, costimulated. Visual cortex displays postcritical period ocular dominance stripes and the functional property of stereopsis. Somatosensory cortex displays segregated domains of dorsal and glabrous representation that remain plastic into adult life but whose functional relevance is currently unknown.

This comparison sharpens the question of the functional importance of plastic changes in cortical maps. From the viewpoint of a selective theory, the divergent and overlapped nature of the anatomy is an unavoidable result of development which involves dynamic processes of cell adhesion, movement, and death, rather than prespecified molecular markers (Edelman, 1986). Given these developmental constraints, a dynamic selective process is required to create precise functional maps. The observed map plasticity is then an ongoing manifestation of the existence of somatic neural selection. Over evolutionary time scales, this somatic selection could generate well-adapted functional maps despite the changing anatomical substrates (such as the growth of existing areas or the addition of new areas). During the lifetime of an animal, plasticity in functional maps ensures that magnification factors reflect recent usage, ensuring the devotion of finer discriminitive abilities (as reflected by smaller receptive fields) to more frequently used peripheral sites. Competitive plasticity also maximizes the use of cortical representation space, ensuring that large areas are not devoted to nonadaptive representations. A final possibility has to do with what Mountcastle (1984) has termed "parallel and serial processing" in somesthesis: The reentrant linking of representations in other cortical and subcortical areas may be facilitated by the ability of each individual representation to undergo plastic changes in location or modality.

Although the present model does not address the develop- 
mental aspects of the formation of topographic projections, it may, ncverthcless, bc of somc relcvance to the organization of such projections, particularly in cases where there is dynamic, activity-dependent reorganization such as in the retinotectal projection (Schmidt, 1985; Fraser and Hunt, 1986). In contrast, the model may not apply strictly to certain specialized areas in which map borders are fixed by the anatomy itself. This occurs in the rodent whisker barrel fields (Woolsey and Van der Loos, 1970; Killackey, 1973), and possibly in the somatosensory cortex of the raccoon (Warren and Pubols, 1984; Rasmusson and Nance, 1986). Even in these cases, however, the assumptions of the model may apply to map changes at the level of the thalamus, dorsal column nuclei, or spinal cord. There is substantial evidence for such subcortical map changes (Wall and Eggers, 1971; Dostrovsky et al., 1976; Devor and Wall, 1981), and we would expect that they play an important role in the overall mapping changes observed at the cortical level. Due to the convergence and divergence of fibers, local shifts at the dorsal column or thalamic level could give rise to much larger shifts at the cortical level. In addition, the reentrant (Edelman, 1981) nature of these projections (the presence of substantial reciprocal projections which send phasically coordinated signals back to earlier levels) implies that map changes at the different levels will closely correspond. The network considerations addressed here could be extended to the modeling of these situations.

It is useful, finally, to present a number of predictions prompted by the results of the simulations:

1. Neuronal groups as defined in this study will be found in the cortex.

2. Abolition of shunting inhibition (through chloride channel blockers such as $\mathrm{Zn}^{2+}$ ) should generally lead to greater network instabilities than abolition of hyperpolarizing inhibition. The effects of the actions of barbiturates, benzodiazepines, and specific toxins such as bicuculline and picrotoxin on GABA-receptor-associated chloride channels (Olsen, 1987) may be related to the network instabilities observed here.

3. The minimum extent for segregated zones of like modality in a map is one group diameter. Statistical analyses of the size distribution of segregated areas in cortical maps might reveal the presence of a discrete subunit size. There should be 2 distinct classes of map border shifts: large shifts that occur over distances representing an integral number of groups and much smaller local shifts due to the exchange of individual cells between groups. Besides the results reported here, other reported experiments such as digit amputations, or local cortical ablations, will be accounted for in further tests of the model.

4. If 2 adjacent digits are independently stimulated for prolonged periods of time, the representation of each would expand in all directions except along their common border.

5. Cells with mixed glabrous and dorsal receptive fields may be found at the borders of neuronal groups, particularly between dorsal and glabrous groups, and in higher density at the edges of a cytoarchitectonic region.

6. Concurrent stimulation of a glabrous and a corresponding dorsal region should lead to a group with a mixed receptive field. If repeated for a prolonged time, the previously segregated representations may be subsumed into a new conjoint representation. It would then be of interest whether in a psychophysical experiment, stimulation of just the glabrous or just the dorsal surface of the region could be subjectively localized in an appropriate manner. In other words, does the perception of the location of a stimulus depend upon unique cortical loci for the representation of that location, or can it be independently identified, for example, from the firing properties of different modalities or comparisons between multiple representations?

The network simulations presented here are based on a general synthetic approach to neural modeling in which experimental phenomena observed at several different structural and physiological levels are simulated together in sufficiently powerful computers using simplified but realistic assumptions. Given an understanding of such minimal models, we suggest that this approach may be valuable in understanding other dynamic processes of selection and mapping in complex nervous systems. It will be of particular interest, for example, to determine the effects of adding additional synaptic stages between the receptor sheet and the "cortex" and also to incorporate a laminated cortex with layer-specific inputs and outputs.

\section{References}

Daniel, P. M., and D. Whitteridge (1961) The representation of the visual field in the cerebral cortex in monkeys. J. Physiol. (Lond.) 159: 302-321.

Devor, M., and P. D. Wall (1981) Effects of peripheral nerve injury on receptive fields of cells in the cat spinal cord. J. Comp. Neurol. 199: 227-291.

Dostrovsky, J. O., J. Millar, and P. D. Wall (1976) The immediate shift of afferent drive of dorsal column nucleus cells following deafferentation: A comparison of acute and chronic deafferentation in gracile nucleus and spinal cord. Exp. Neurol. 52: 480-495.

Dykes, R. W. (1983) Parallel processing of somatosensory information: A theory. Br. Res. Rev. 6: 47-115.

Edelman, G. M. (1978) Group selection and phasic reentrant signalling: A theory of higher brain function. In The Mindful Brain, G. M. Edelman and V. B. Mountcastle, eds., pp. 51-100, MIT Press, Cambridge, MA.

Edelman, G. M. (1981) Group selection as the basis for higher brain function. In Organization of the Cerebral Cortex, F. O. Schmit, F. G. Worden, G. Adelman, and S. G. Dennis, eds., pp. 535-563, MIT Press, Cambridge, MA.

Edelman, G. M., and L. H. Finkel (1984) Neuronal group selection in the cerebral cortex. In Dynamic Aspects of Neocortical Function, G. M. Edelman, W. E. Gall, and W. M. Cowan, eds., pp. 653-695, Wiley, New York.

Finkel, L. H. (1985) Selection in synaptic regulation and neural mapping. In Molecular Basis of Animal Form (Proc. of UCLA Symposium), G. M. Edelman, ed., pp. 571-618, Alan R. Liss, New York.

Finkel, L. H., and G. M. Edelman (1985) Interaction of synaptic modification rules within populations of neurons. Proc. Natl. Acad. Sci. USA 82: 1291-1295.

Finkel, L. H., and G. M. Edelman (1987) Population rules for synapses in networks. In Synaptic Function, G. M. Edelman, W. E. Gall, and W. M. Cowan, eds., pp. 711-757, Wiley, New York.

Fraser, S. E., and R. K. Hunt (1986) A physiological measure of shifting connections in the Rana-pipiens retinotectal system. J. Embryol. Exp. Morphol. 94: 149-161.

Gilbert, C. D., and T. N. Wiesel (1979) Morphology and intracortical projections of functionally characterized neurons in the cat visual cortex. Nature 280: 120-125.

Hawkins, R. D., T. W. Abrams, T. J. Carew, and E. R. Kandel (1983) A cellular mechanism of classical conditioning in Aplysia-Activity dependent amplification of pre-synaptic facilitation. Science 219:400405.

Hebb, D. O. (1949) The Organization of Behavior, Wiley, New York.

Huganir, R. L., A. H. Delacour, P. Greengard, and G. P. Hess (1986) Phosphorylation of the nicotinic acetylcholine receptor regulates its rate of desensitization. Nature 321: 774-776.

Ito, M., M. Sakurai, and P. Tongroach (1982) Climbing fiber-induced depression of both mossy fiber responsiveness and glutamate sensitivity of cerebellar Purkinje cells. J. Physiol. (Lond.) 324: 113-134.

Jenkins, W. M., M. M. Merzenich, and M. T. Ochs (1984) Behaviorally controlled differential use of restricted hand surfaces induce changes 
in the cortical representation of the hand in area $3 \mathrm{~b}$ of adult owl monkeys. Soc. Neurosci. Abstr. 10:665.

Kaas, J. H., M. M. Merzenich, and H. P. Killackey (1983) The reorganization of somatosensory cortex following peripheral-nerve damage in adult and developing mammals. Annu. Rev. Neurosci. 6: 325-356.

Kelso, S. R., A. H. Ganong, and T. H. Brown (1986) Hebbian synapses in hippocampus. Proc. Natl. Acad. Sci. USA 83: 5326-5330.

Killackey, H. P. (1973) Anatomical evidence for cortical subdivisions based on vertically discrete thalamic projections from the ventral posterior nucleus to cortical barrels in the rat. Brain Res. 51: 326331.

Landry, P., and M. Deschenes (1981) Intracortical arborizations and receptive fields of identified ventrobasal thalamocortical afferents to the primary somatic sensory cortex in the cat. J. Comp. Neurol. 199: 345-371.

Leyton, A. S. F., and C. S. Sherrington (1917) Observations on the excitable cortex of the chimpanzee, orangutan, and gorilla. Q. J. Exp. Physiol. 11: 137-222.

Malinow, R., and J. P. Miller (1986) Postsynaptic hyperpolarization during conditioning reversibly blocks induction of long-term potentiation. Nature 320: 529-530.

Meinhardt, H. (1982) Models of Biological Pattern Formation, Academic, New York.

Merzenich, M. M., J. H. Kaas, M. Sur, and C. S. Lin (1978) Double representation of the body surface within cytoarchitectonic Areas $3 \mathrm{~b}$ and 1 in "S-I" in the owl monkey Aotus trivirgatus. J. Comp. Neurol. 181: 41-74.

Merzenich, M. M., J. H. Kaas, J. T. Wall, R. J. Nelson, M. Sur, and D. J. Felleman (1983a) Topographic reorganization of somalosensory cortical areas $3 \mathrm{~b}$ and 1 in adult monkeys following restricted deafferentation. Neuroscience 8: 33-55.

Merzenich, M. M., J. H. Kaas, J. T. Wall, R. J. Nelson, M. Sur, and D. J. Felleman (1983b) Progression of change following median nerve section in the cortical representation of the hand in areas $3 \mathrm{~b}$ and 1 in adult owl and squirrel monkeys. Neuroscience 10:639-665.

Merzenich, M. M., R. J. Nelson, M. P. Stryker, M. Cynader, A. Schoppman, and J. M. Zook (1984) Somatosensory cortical map changes following digit amputation in adult monkeys. J. Comp. Neurol. 224: 591-605.

Mountcastle, V. B. (1957) Modality and topographic properties of single neurons of cat's somatic sensory cortex. J. Neurophysiol. 20 408-434.

Mountcastle, V. B. (1984) Central nervous mechanisms in mechanoreceptive sensibility. In Handbook of Physiology, Sect. 1: The Nervous System, Vol. 3, pp. 789-878, American Physiological Society, Bethesda, MD.

Olsen, R. W. (1987) The $\gamma$-aminobutyric acid/benzodiazepine/barbituate receptor-chloride ion channel complex of mammalian brain. In Synaptic Function, G. M. Edelman, W. E. Gall, and W. M. Cowan, eds., pp. 257-271, Wiley, New York.

Rasmusson, D. D., and D. M. Nance (1986) Non-overlapping thalamocortical projections for separate forepaw digits before and after cortical reorganization in the racoon. Brain Res. Bull. 16: 399-406.

Reeke, G. N., Jr. (1984) The ROCKS System of computer programs for macromolecular crystallography. J. Appl. Crystallogr. 17: 125130.

Schmidt, J. T. (1985) Formation of retinotopic connections-selective stabilization by an activity-dependent mechanism. Cell. Mol. Neurobiol. 5: 65-84.

Sur, M., M. M. Merzenich, and J. H. Kaas (1980) Magnification, receptive field area and 'hypercolumn' size in areas $3 \mathrm{~b}$ and 1 of somatosensory cortex in owl monkeys. J. Neurophysiol. 44: 295-311.

Turing, A. (1952) The chemical basis of morphogenesis. Phil. Trans. R. Soc. B 237: 37-72

Wall, P. D., and M. D. Eggers (1971) Formation of new connections in adult rat brains after partial deafferentation. Nature 232: 542-544.

Warren, S., and B. H. Pubols (1984) Somatosensory thalamocortical connections in the racoon: An HRP study. J. Comp. Neurol. 227: 597-606.

Whitsel, B. L., and D. G. Kelly (1986) Knowledge acquisition ("learning") by the somatosensory cortex. In Brain Structure and Function. E. G. Wegman, J. Davis, and R. Newburgh, eds., AAAS Neuroscience Symposium.

Wigstrom, H., and B. Gustafsson (1983) Heterosynaptic modulation of homosynaptic long-lasting potentiation in the hippocampal slice. Acta Physiol. Scand. 119: 455-458.

Woolsey, T. A., and H. Van der Loos (1970) The structural organization of layer IV in the somatosensory region (SI) of mouse cerebral cortex. The description of a cortical field composed of discrete cytoarchitectonic units. Brain Res. 27: 205-242. 